xenograft tumor model was introduced to investigate the role of OLA1 in vivo.

Results OLA1 demonstrated a higher expression in gastric cancer tissues with stage III-IV compared to paired normal tissue (25 paired sample; $P<0.001$ ), and the high level of OLA1 was correlated with poor prognosis $(\mathrm{HR}=2.40, P=0.008)$ and decreased sensitivity to 5-fluorouracil adjuvant chemotherapy $(\mathrm{HR}=2.63, P<0.001)$. Overexpression of OLA1 in gastric cancer cells reduced the sensitivity to 5-fluorouracil treatment, while knockdown of OLA1 by shRNA in gastric cancer cells significantly inhibited the cell proliferation, migration, invasion and tumorigenicity. However, OLA1 did not affect the cell cycle and proliferation in normal gastric epithelium cells. Mechanistically, WGCNA analysis of TCGA RNA-seq data suggested that high expression of OLA1 will promote $\mathrm{G} 2 / \mathrm{M}$ phase transition and eventually lead to tumor progression, while knockdown of OLA1 in gastric cancer cells significantly prolonged $\mathrm{G} 2 / \mathrm{M}$ phase (control vs $\mathrm{KD}$ : $10.65 \%$ vs $21.59 \%$; $P<0.01)$.

Conclusions OLA1 inhibition could suppress gastric cancer progression and enhance the sensitivity to adjuvant chemotherapy with sparing normal gastric epithelium cells. Therefore, OLA1 could be a novel and promising target in treating gastric cancer.

\section{IDDF2021-ABS-0110 THE FEASIBILITY OF PERCUTANEOUS ENDOSCOPIC GASTROSTOMY (PEG) IN POST-GASTRECTOMY PATIENTS}

Toh Yoon Ezekiel Wong ${ }^{*}$, Kaori Yoneda. Hiroshima Kyoritsu Hospital, Japan

\subsection{6/gutjnl-2021-IDDF.140}

Background Although percutaneous endoscopic gastrostomy (PEG) is considered the route of choice for long-term enteral nutrition, this procedure may be difficult to perform in postgastrectomy patients and research addressing this issue is limited. We report our experience with PEG, percutaneous endoscopic duodenostomy (PED) and percutaneous endoscopic jejunostomy (PEJ) performed in post-gastrectomy patients at our hospital.

Methods 29 post-gastrectomy patients (23 men and 6 women) who received PEG for enteral nutrition at our hospital between April 2007 and April 2021 were included in this study. Baseline characteristics and clinical outcomes of interest were analyzed retrospectively.

Results Mean age was 81.7 (range: 61 to 93) years old. 18 patients (62\%) had cerebrovascular disease, 14 patients $(48 \%)$ had dementia and 12 patients (41\%) had respiratory diseases as comorbidities contributing to their dysphagia. 22 patients underwent distal gastrectomy (14 Billroth I procedures and 8 Billroth II procedures), 2 patients received proximal gastrectomy and 5 had either wedge or central gastrectomy. 26 patients received their catheter placements using the Push technique, while 3 had the Introducer technique used. In 8 patients with distal gastrectomy, the catheter was inserted slightly beyond the anastomosis site (PED/PEJ). Postoperatively, 8 patients $(28 \%)$ developed peristomal infection/leakage, including 3 of the PED/PEJ patients (37\%). Feeding-related aspiration pneumonia was observed in 5 patients $(17 \%)$ and diarrhea in 3 patients (10\%). The average postoperative length of stay was 25.6 days. 3 patients $(10 \%)$ died before discharge but none within 30 days after the procedure.
Conclusions We reported our experience with PEG/PED/PEJ in 29 post-gastrectomy patients. When successfully performed in these patients, PEG can remain a useful procedure for enteral nutrition, with mortality rates comparable to other PEG patients. However, post-gastrectomy patients (especially for $\mathrm{PED} / \mathrm{PEJ}$ ) may be more prone to peristomal complications.

\section{IDDF2021-ABS-0111 NOMOGRAM OF DEVELOPMENT AND VALIDATION FOR ESTIMATION OF INADEQUATE BOWEL PREPARATION RISK BEFORE COLONOSCOPY IN ADULTS}

${ }^{1}$ Xin Yuan*, ${ }^{1}$ Hui Gao, ${ }^{1}$ Shuhao Zheng, ${ }^{2}$ Cenqin Liu, ${ }^{3}$ Weihong Wang, ${ }^{3}$ Jiarong Xie, ${ }^{3}$ Zhixin Zhang, ${ }^{3}$ Lei Xu. ${ }^{1}$ School of Medicine, Ningbo University, China; ${ }^{2}$ School of Medicine, Zhejiang University, China; ${ }^{3}$ Department of Gastroenterology, Ningbo First Hospital, China

\subsection{6/gutjnl-2021-IDDF.141}

Background To develop and validate an updated nomogram of inadequate bowel preparation risk prediction in adults before colonoscopy.

Methods 1,216 underwent colonoscopy in a tertiary hospital in China: 916 patients in the derivation cohort were used to develop the nomogram and a separate set of 300 patients in the validation cohort were used to validate the model. Patients were ready for bowel preparation before colonoscopy. Logistic regression model in the derivation cohort to develop the nomogram. Risk factors considered included age, gender, education level (illiteracy or primary, junior high, senior high and college or higher), hospitalization, body mass index (BMI), diabetes, hypertension, history of surgery (colorectal surgery, other abdominal/pelvis surgery and no surgery), constipation, examination time (morning or afternoon), history of colonoscopy [history of adequate bowel preparation (ABP), history of inadequate bowel preparation (IBP) and no history of colonoscopy], indication of colonoscopy (diagnosis, physical examination and surveillance) and medication (use drug or not). Measures of discrimination and calibration were determined in the internal and external validation cohort separately. Inadequate bowel preparation defined by Boston Bowel Preparation Score (BBPS) with less than 6 in total or less than 2 in any segment colon.

Results 224 patients of inadequate bowel preparation (24.5\%) were identified in the derivation cohort. Only 5 risk factors were considered in the final model (IDDF2021-ABS-0111

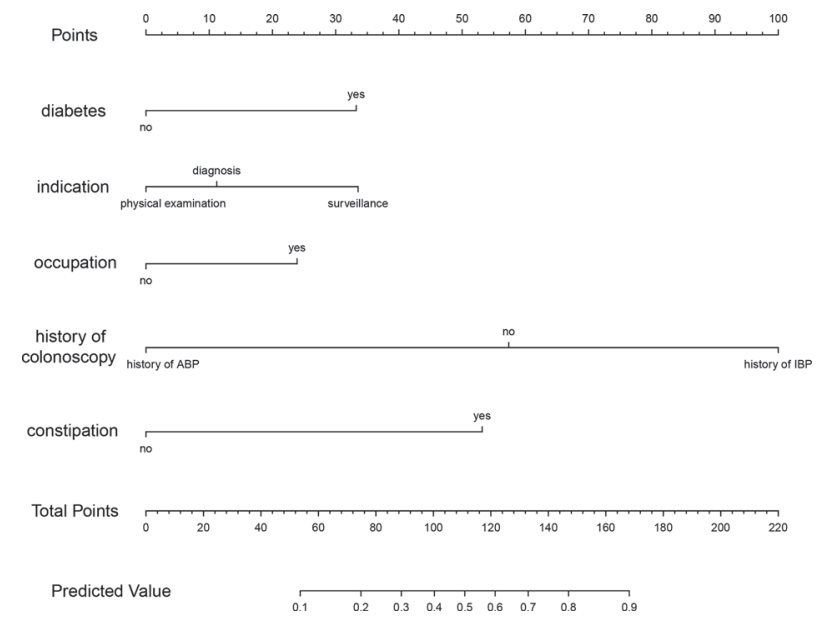

Abstract IDDF2021-ABS-0111 Figure 1 